\title{
Museos y jóvenes: entre la incomprensión y el desencanto. Percepciones y argumentos juveniles sobre el consumo cultural de museos en Chile
}

\author{
Museums and youth: between misunderstanding and \\ disappointment. Youth perceptions and arguments about \\ the cultural consumption of museums in Chile
}

\author{
Cristian David Antoine-Faúndez \\ Universidad del Pacífico. Santiago, Chile. \\ cantoine@upacifico.cl \\ JAVIERA CARMONA-JimÉNEZ \\ Universidad de Playa Ancha. Valparaíso, Chile. \\ javiera.carmona@upla.cl
}

Recibido: 1 de marzo de 2014

Aprobado: 7 de mayo de 2014

\section{Resumen}

Los jóvenes son el público de museos menos explorado en los estudios sobre audiencias museales, situación paradójica al considerar su lugar estratégico en la reproducción cultural y el alto consumo cultural que emprenden en comparación con el resto de los sectores. El fenómeno del consumo de museos por jóvenes chilenos que se auto reconocen como público y no-público de museos se explora a partir de una aproximación cualitativa realizada con focus groups en las tres ciudades más grandes de Chile (Santiago, Valparaíso y Concepción). Entre las percepciones de los y las participantes se identifica la valoración del museo como institución cultural plenamente vigente, sin embargo en el cuestionamiento a la actividad de los museos los jóvenes revelan la especificidad de su matriz cultural, referida a una temporalidad social basada en el fragmento, el discurso de la familiaridad, proximidad y a la vez de la ruptura y crítica, y la experiencia estética/histórica museal basada en el placer y la fruición. Se propone una perspectiva general para avanzar en el esclarecimiento de los consumos culturales juveniles que permitan caracterizar de manera más precisa el lugar del museo y así diseñar políticas museales más eficaces.

Palabras clave: museos, jóvenes, consumo cultural, público de museos, no público de museos.

Antoine-Faúndez, C. D., Carmona-Jiménez, J. (2015): Museos y jóvenes: entre la incomprensión y el desencanto. Percepciones y argumentos juveniles sobre el consumo cultural de museos en Chile. Arte, Individuo y Sociedad, 27(2) 227-242 


\begin{abstract}
Young people are less explored in museum audience research; this is a paradoxical situation when considering its strategic location in the cultural reproduction and if considering the high performing cultural consumption compared with other sectors. The phenomenon of museums consumption by young Chileans who are self recognized as public and non-public museums is explored from a qualitative approach. It was conducted with focus groups in the three largest cities in Chile (Santiago, Valparaíso and Concepción). They identify the museum as a cultural institution in full force. However, in questioning museums activity youth reveal the specificity of their cultural matrix. This is referred to a social temporality based on the fragment, the discourse of familiarity, proximity and instead of breaking and critical. They claim a museum aesthetic / historical experience based on pleasure and enjoyment. An overview is proposed to further clarify the youth cultural consumption to characterize more precisely the place of the museum in the set, to design more effective policies museums. Keywords: museum, youth, cultural consumption, museum visitors, museum non-visitors.
\end{abstract}

Sumario: 1. Consumo cultural de museos: realidades heterogéneas y complejas relaciones, 2. Jóvenes, consumo cultural y museal en Chile: visiones contrarias, 3. Jóvenes y museos: desencanto e incomprensión, 4. Iniciativas de los museo para aproximarse a los jóvenes 5. Zapping y tiempo detenido: encuentros/desencuentros de los jóvenes con el museo, 6. Conclusiones. Referencias.

Este artículo recoge resultados del proyecto de investigación "Los museos y su público. Evaluación y Análisis de la comunicación y la gestión de audiencias museísticas en Chile 2012-2014, Fondecyt Regular N¹120313”, financiado por el Consejo Nacional de Investigación Científica y Tecnológica (CONICYT) de Chile.

\title{
1. Consumo cultural de museos: realidades heterogéneas y complejas relaciones
}

El acceso de la población a los museos suele ser una de las categorías incluidas con frecuencia en los estudios sobre consumo cultural (García Canclini 1993; Bisbal, 1999; Bouder-Pailler \& Damak, 2009). Este último se refiere a los distintos tipos de apropiación de aquellos bienes cuyo principal valor percibido es el simbólico. Producidos y consumidos en circuitos relativamente diferenciados para su uso y apropiación, los consumidores se distinguen por poder adquisitivo de acuerdo a los diferentes grupos sociales y a las disparidades en educación y capital cultural (Bourdieu, 1988; Chan \& Goldthorpe, 2010; Sunkel 2002). Es esta perspectiva socioantropológica la que matiza el origen económico del concepto "consumo cultural", y lo concibe como práctica social (e individual) de apropiación, vivencia y uso de bienes y servicios culturales disponibles en la sociedad, es lo que genera un dinamismo en los esquemas simbólicos y de percepción de los sujetos, renovando horizontes de expectativas sociales y abriendo nuevos planos de desarrollo social, económico y humano (Appadurai, 1988; McCracken 1990; Douglas \& Isherwood, 1996; Certeau, 1996; Barbero, 1999).

La comprensión de los variados mecanismos que participan en la demanda cultural, entendida como las decisiones individuales de las audiencias que determinan las opciones de consumo de bienes culturales ante una oferta cada vez más heterogénea, permite diseñar y evaluar políticas culturales destinadas a una totalidad social pero a la vez sensibles a las oscilaciones de la interacción entre lo que produce una sociedad 
y los modos de uso y apropiación de tales bienes (Boylan, 2006). En definitiva, los estudios de audiencias registran las demandas, necesidades, patrones de percepción y gusto de los públicos (Rosas Mantecón, 2005).

Trabajos recientes sobre el consumo cultural que representan los museos en México, por ejemplo, revelan el interés permanente por esclarecer las características del acceso de la población a estas instituciones culturales, no obstante se critica el carácter meramente descriptivo que tienen las encuestas (Almanza, 2005) insuficientes para explicar el complejo fenómeno sociocultural de consumo museal. También hay estudios disponibles para el caso argentino (Cousillas, Altamirano, Cardinal, Di Fiore, \& Ricci, 1998; Bialogorski \& Cousillas, 2008) y el español (Laboratorio Permanente de Públicos de Museos, 2010) orientados por la misma línea cuantitativa descriptiva. Para el caso chileno, las estadísticas de acceso a los museos, en el contexto del consumo cultural de la población, están consignadas en el Informe Anual Cultura y Tiempo Libre 2003-2009 (INE, 2010) y en la Encuesta Nacional de Participación y Consumo Cultural (CNCA, 2012). Las referencias sobre el público asistente a museos o visitantes se expresa por lo general en datos estadísticos, lo que revela la persistencia de la tendencia latinoamericana avizorada en 1990 hacia la investigación empírica que dejó en un lugar secundario la reflexión teórica (Rosas Mantecón 2005).

En Latinoamérica se sabe poco del público de los museos, pero se sabe menos del que no asiste a los museos. La categoría de análisis del no-público es, de entrada, también compleja, y abarca situaciones muy distintas. Algunos especialistas (Ancel \& Pessin, 2004; Mengin, 1993; Asensio \& Asenjo Vergara, 2011) plantean la distinción entre visitantes potenciales y no-público, considerando a los primeros como aquellas personas que no han utilizado los museos pero para las cuales no existen barreras suficientemente importantes que puedan impedirles esta práctica en un futuro. El no-público de museos comprende entonces a las personas para las que existen barreras de exclusión originadas por múltiples factores, que les impiden utilizar los museos. Estas trabas no se reducen a la accesibilidad física y sensorial, la que ya segrega entre "público en general" y "personas con necesidades especiales", como de aquellas que devienen del diseño universal del museo (De la Iglesia \& Roselló, 2014). Los obstáculos que caracterizan al no-público (Kirchberg, 1996) se refieren a la accesibilidad conceptual, situación especialmente delicada en espacios en los que el mundo de los significados cobra especial relevancia, como los museos de arte. La distinción basada en una oferta general para visitantes-tipo y otra opción para los que están fuera de ese patrón se vuelve inútil, pues incluso parte importante del público puede estar excluido de disfrutar de la oferta museal (Schouten, 1998; Gómez del Águila, 2011).

Son los jóvenes el público de museos más inexplorado de acuerdo a la literatura disponible (Lekue, 2009), dato paradójico si se considera que es el sector de la población que mayor consumo cultural emprende a nivel mundial (Stuedahl, 2011).

En el presente artículo se explora el fenómeno del consumo y no-consumo de museos por jóvenes en Chile a partir de una aproximación cualitativa basada en la realización de grupos focales en las tres ciudades más importantes de Chile (Santiago, Valparaíso y Concepción). A partir de este método de recolección de datos se exponen el conjunto de percepciones de los jóvenes público y no-público sobre los museos y el 
modo en que éstas influyen en su decisión de visitar/no visitar museos. Se identificó la concepción que este grupo ha construido sobre el museo como institución en relación a la función social que según ellos cumplen, el sentido que le atribuyen a una visita, y la experiencia museal que demandan. La finalidad de esta aproximación fue introducir una mirada complementaria a la discusión sobre audiencias museales al centrar, para efectos de este artículo, la atención en un enfoque cualitativo del consumo cultural juvenil en Chile (Bermúdez, 2001; Barbero \& Morduchowicz, 2008). La información contenida en esta presentación corresponde así a una porción de un estudio mayor titulado "Los museos y su público. Evaluación y análisis de la comunicación y gestión de las audiencias museísticas en Chile (2012-2014)", en el que los autores, principalmente académicos de diferentes escuelas de Periodismo y directores de museos, participaron en la construcción de un diagnóstico general sobre las estrategias de comunicación y gestión de las audiencias de los museos en Chile. Entre las conclusiones del trabajo global se destaca la gran variedad de concepciones entre los museos sobre sus públicos y su función comunicativa, lo que deriva en una dispersión de prácticas no articuladas, carentes de una estrategia integral de gestión de sus audiencias, lo que es especialmente notorio en la relación con los jóvenes.

\section{Jóvenes, consumo cultural y museal en Chile: visiones contrarias}

Según la Dirección de Bibliotecas Archivos y Museos de Chile (DIBAM), durante el 2012 se cortaron 1.877.136 entradas entre los 26 museos que administra (23 regionales y 3 nacionales). Esta cifra equivale a 304 mil más que los visitantes del 2009, y pese al aumento que implica el número de usuarios de los museos estatales en Chile, lo cierto es que la cifra de asistentes se ha mantenido más o menos constante desde el 2007. Para el organismo estatal que dirige los museos públicos en Chile, el promedio de 1.400 .000 visitantes a sus museos estatales es satisfactorio pues sostienen que equivale casi al $10 \%$ de la población chilena, de manera que al extender esta proporción a los 211 museos del país que pertenecen a diversas entidades (universidades, fuerzas armadas, privados, etc.) sería un $20 \%$ de la población la que asiste a los museos, situación "más que razonable" (RPC 2005: 30). Los estudios disponibles sobre el consumo museal de los jóvenes chilenos, la mayoría de corte cuantitativo, anuncian un escenario desfavorable en las políticas culturales, especialmente de aquellas orientadas al desarrollo de audiencias (Antoine, 2012). El museo en su mirada cotidiana no estaría siendo reconocido por los jóvenes chilenos como espacio que reúne y explicite lo colectivo; tampoco respondería a la dimensión socializadora que reclaman, y por último, no se les advertiría como espacio de lo íntimo, de la memoria y del porvenir (Observatorio Cultural, 2011).

El consumo museal en Chile tiene cuatro características fundamentales al examinarse el cruce correlacional entre cantidad de audiencia y variables como grupos etarios, nivel socioeconómico, emplazamiento geográfico, como capital o provincia (Observatorio Cultural, 2011). La primera es que el 73,8\% de la población chilena no asiste a museos (ENPCC 2012: 43); en segundo lugar se confirma la asociación histórica entre cantidad de visitantes y estratificación social, así los niveles socioeconómicos altos acuden más frecuentemente al museo, a diferencia de los más bajos que no alcanza el 5\% de asistencia. Las estadísticas corroboran además 
la preferencia por los museos de arte entre los sectores de altos ingresos, mientras que la clase media y sectores bajos se inclinan por los museos históricos (Observatorio Cultural, 2011: 14). Por último, los mayores índices de visitas a museos no están en la capital, donde se concentra la mayor y variada oferta museal, sino en dos ciudades ubicadas en los extremos norte y sur del país, donde la oferta museal es en extremo limitada y se reduce a dos instalaciones en cada localidad (Antofagasta y Magallanes).

Las cifras reportadas por las Encuestas de Participación y Consumo Cultural sobre la asistencia a museos reflejan el itinerario de las prácticas culturales por el que han circulado los chilenos y chilenas en las últimas décadas. En esta tendencia general, la radiografía del público asistente a museos no revela grandes sorpresas: un público que se muestra invariable en cuatro años $(20,8 \%$ de los encuestados declara haber asistido a lo menos una vez en el último año), homogéneo entre hombres y mujeres, principalmente joven (cerca de la mitad se ubica entre los 15 y 44 años), y que representa en su mayoría a los sectores acomodados del país (39,8\% de los asistentes pertenecen al nivel socioeconómico $\mathrm{ABC} 1$ ). En las cifras señaladas destaca la existencia de una distancia importante entre los museos y las prácticas e intereses de la mayoría de los chilenos. En efecto, los asistentes pertenecen mayoritariamente al mismo reducido segmento social y etario que se observa en otros ámbitos culturales, y aquellos que no asisten, argumentan con la falta de tiempo o la ausencia de interés. Dicho de otro modo, el museo no forma parte de las prioridades de los chilenos a la hora de gozar de la cultura (Bastías Sekulovic, 2012).

Son los jóvenes de 15 a 29 años el grupo etario de mayor consumo cultural en Chile, por ello no sorprende que sea el que además declara mayor frecuencia de visita a museos (CNCA 2011:18). Sin embargo, en sus prácticas de consumo cultural existe una gran diferencia entre la visita a los museos y el resto de su consumo (Gayo, 2013). Este delta es menor en los otros grupos etarios, de tal manera que en los adultos divididos en diversos rangos, la poca frecuencia de visitas a museos corresponde a un consumo cultural bajo en general. No hay duda que la edad influye casi tanto como la clase en los gustos y prácticas culturales en general (Savage \& Gayo 2011; Bennett, 2009). El estudio del consumo cultural de los jóvenes tiene por primera dificultad superar las limitaciones de la propia categoría social de "jóvenes", la que ya anula la diversidad de lo que abarca y favorece la aproximación desde miradas unitarias o generalizadoras.

Tanto las mediaciones que hace el CNCA y el Instituto Nacional de Estadísticas (INE) confirman que los asistentes a museos que aparecen tabulados parten con la categoría "Menores" que incluye a todos los visitantes de menos de 18 años. Las encuestas se realizan entrevistando en los hogares a una persona seleccionada al azar, con la condición de que esa persona tuviera 15 años o más. Por eso los tabulados parten con la categoría de 15 a 29 años. Este detalle en la medición de los menores de edad es probablemente la razón de la diferencia entre los sondeos generales y las encuestas de cada museo, porque estas instituciones, durante los últimos años, han invertido una gran cantidad de recursos humanos y financieros en potenciar sus áreas educativas y éstas a su vez, han centrado sus esfuerzos en invitar y motivar la participación de niños y escolares, y es en estos segmentos precisos que los museos presentan mayores aumentos de visitantes (Cari, 2013). Contrastar el enfoque 
estadístico con una aproximación cualitativa nos perece podría contribuir, al menos preliminarmente, a establecer una mirada más integradora de las motivaciones de las audiencias que asisten a estos espacios culturales.

\section{Jóvenes y museos: desencanto e incomprensión}

Sobre el público juvenil y su relación con los museos predomina la opinión que sustenta la decepción ("a los jóvenes les aburre ir al museo"), seguida por el supuesto ideológico que se instala como certeza ("ir al museo los hará más cultos"), por sobre investigaciones que esclarezcan de manera más precisa la relación de este público hacia los objetos, prácticas e instituciones de la cultura, como libros, participación cultural y museos, por mencionar alguno. El debate en torno a la "desafección museal" de los jóvenes se asemeja en parte al diagnóstico catastrofista sobre la crisis de la lectura, cuestión que pese a su importancia en la transmisión de conocimiento y su lugar prioritario en las política educacionales, rara vez se aborda como objeto de estudio (Poulain 2004). Lo mismo ocurre con el debate sobre los jóvenes y su relación con los museos, aspecto escasamente investigado. Es el conocimiento reciente disponible sobre las transformaciones en las prácticas culturales de los jóvenes referidas a la lectura lo que motivó a algunos a renunciar a la desazón y preguntarse si efectivamente existe una crisis de lectura y del libro entre las generaciones más jóvenes (Baudelot, Cartier \& Détrez, 2010; Bazin, 2005; Domínguez \& Sádaba, 2005). Al parecer, el orden de la lectura, entendido como una experiencia construida por la cultura escrita occidental, estaría en disolución por la prescindencia del escrito tradicional ante nuevas formas de circulación del saber y percepciones sobre el gusto, expresado en los síntomas de una metamorfosis del conjunto de hábitos de apropiación y uso.

Al seguir esta argumentación se podría suponer que el orden de "la experiencia museal" vive también los efectos de la auténtica revolución de los comportamientos culturales de las masas, con nuevas prácticas de uso y apropiación de los bienes y servicios culturales a través de las nuevas tecnologías, lo que influye en el papel y presencia del museo en la sociedad contemporánea. El presunto desencanto que se le atribuye a los jóvenes por el museo encubriría más bien la inadvertencia o incomprensión de las tradicionales instituciones de la modernidad occidental (museo, escuela) sobre las prácticas de estos actores sociales, los mejor dotados para asumir la irreversibilidad de los cambios socio-culturales que impone la internacionalización, nuevas formas de conocimiento y el desarrollo tecnológico (Reguillo 2000).

En términos generales el consumo cultural juvenil es fragmentado y heterodoxo (Storey, 1999). En simultáneo ostentan prácticas homogéneas y a la vez versátiles de acceso y apropiación a ciertos bienes culturales y servicios de acuerdo a las identidades personales y distinciones colectivas derivadas del consumo (Kumar Warikoo, 2011). El consumo cultural de los jóvenes chilenos se inscribe en la macro tendencia a nivel latinoamericano que si bien reconoce el peso de las condicionantes económicas, educativas y etarias en la apropiación y uso de los bienes culturales (Rey, 2008), el incremento en la disponibilidad de estos recursos junto con el aumento de la individuación de las personas, deja al descubierto nuevos factores (PNUD, 2002; Güell, Morales \& Peters, 2011). Para Chile se ha establecido que las propias dinámicas del consumo producirían de manera performativa sus propias distinciones, 
lo que convierte a la dimensión socioeconómica en variable independiente (Catalán 2009; Peters 2010). Asimismo, se reconoce que la segmentación social se produce solamente a partir de variables de consumo y no al revés. No es la estructura social lo que diferencia el consumo cultural de los grupos, sino la diferencia de sus prácticas (Torche 2007, 2010). Ambos estudios revelan que el consumo cultural resulta más complejo de lo que permiten explicar las tres hipótesis establecidas (homología, omnívoros-unívoros e individualización). La segmentación que produce el consumo cultural radicaría en las lógicas al interior del propio campo cultural y no en relación con la estructura de la sociedad que determinan el nivel educativo y los recursos económicos (Catalán 2009: 12). Visto así, en el consumo cultural de los jóvenes se podrían rastrear las huellas de lo que se considera el "hastío postmoderno", evidenciando raíces profundas en un recelo de los jóvenes hacia el principio de totalidad social, del todo homogéneo, de la tradición frente al elogio de la atomización y la heterogeneidad, levantando así su propio discurso de lo cultural, aún poco explorado en sus contradicciones y escasamente reconocido por los museos chilenos.

\section{Iniciativas de los museos para aproximarse a los jóvenes}

Los museos han ido desarrollando numerosas estrategias para acercarse al público juvenil. Se aprecian entre ellas iniciativas integradas entre servicios, contenidos, ventajas asociadas, fórmulas participativas y estrategias comunicacionales adaptadas, replicables en otras latitudes acogiendo las claves socio-culturales locales. No nos proponemos realizar aquí una revisión exhaustiva de las mismas, solo nos remitiremos a señalar que buena parte de las visitas de jóvenes y niños a los museos en Chile, ocurre en el marco de visitas escolares organizadas por profesores como actividad docente. Por esto los museos entienden que sus visitantes mayoritarios y fundamentales son el público escolar (incluyendo a los profesores). Esta convicción ha derivado en que el museo termine siendo la extensión de la sala de clases (RPC 2005:29). La tendencia internacional revela que la trayectoria de casi 150 años de atención preferente del museo a grupos escolares vive desde el año 2000 la apertura a nuevos públicos con otras características y necesidades. Las familias se han convertido en protagonista de los programas educativos de muchos museos, ya no orientados solo al sistema escolar (Obregón Fernández 2011:12), primer gesto de aproximación a los jóvenes.

Las iniciativas más conservadoras de los museos para promover el acceso de los jóvenes ofrecen entradas con descuentos, lejanas de las acciones innovadoras y eficientes de otros museos. A la vanguardia están los museos que diseñan y ejecutan estrategias integrales orientadas a crear y fortalecer un vínculo con los jóvenes basadas no sólo en el reconocimiento de sus necesidades (Devos 2006; Gutiérrez, 2010) sino también en comunicarles a este público "inalcanzable" el auténtico interés del museo en descubrir sus demandas y expectativas, y actuar conforme a ello. Esto último implica la construcción de una relación de sentido entre los jóvenes y el museo, supera el descuento tradicional en la entrada y se asocia a una variedad de ventajas por solo ser joven como guías audiovisuales gratuitas (Metropolitan Museum de Nueva York) y el desarrollo de "esquemas de lealtad" (Jones 2012, Wallace, 2006) al crear categorías específicas con beneficios asociados a su perfil como Young Friends del Philadelphia Museum of Art. 
Las buenas prácticas y experiencias de nexo museo y jóvenes responden a estrategias integrales que comienzan con acciones de vinculación con la familia y la escuela, primer momento de la construcción de relación. Numerosos museos europeos y estadounidenses implementan talleres y visitas familiares, como los madrileños Museo Thyseen-Bornemisza, Museo del Prado, Museo de América y el Museo de Andalucía en Granada por mencionar algunos. Es a través de los programas educativos para las familias donde los jóvenes han sido abordados de manera más sistemática, no como público objetivo, sino en atención a las interacciones particulares que en cada familia se producen y las peculiaridades que la caracterizan como grupo multigeneracional, dadas por las oscilaciones de conocimiento entre padres procedentes de ambientes dispares, como con los hijos de diferentes edades y contextos socioculturales (Obregón Fernández 2011:12).

Establecer las particularidades del público joven, identificar sus necesidades y de acuerdo a ello ofrecer contenidos, servicios y actividades especialmente dirigidos es otra medida emprendida por los museos. Los contenidos se enfocan en estimular la participación de los jóvenes en forjar su propia experiencia estética, identitaria y emocional en el museo, así como interactuar con las obras o piezas de la colección, y los artistas (Fernández Orgaz, 2002). Desde 1988 los museos y galerías de arte del Reino Unido, como el Victoria and Albert Museum (actualmente con el programa Create), fueron pioneros en la construcción de discursos y prácticas que le confieren protagonismo al visitante, especialmente a los jóvenes, tendencia que se ha difundido por numerosos museos europeos (Xanthoudaki 1998). Las propuestas educativas son de carácter interdisciplinario (difundidas muchas de ellas a través de esta misma revista), sintonizan con sus hábitos y gustos y proponen actividades flexibles, accesibles y atractivas que combinan la entretención con el aprendizaje. Museos españoles como el Museo Nacional Centro de Arte Reina Sofía de Madrid desarrollan constantemente programas para jóvenes, como el " $<18$ ". El Museo Artiun en Vitoria ejecuta el programa "A tu medida" con las escuelas del entorno a partir de un tema de interés para los estudiantes (Lekue, 2009). La galería Tate Britain dispone en tanto del Young People's Programmes en el que diseñan variados recursos interpretativos y métodos que privilegian la voz del espectador, introducida y legitimada en el texto de la interpretación de la obra, y se dirige a jóvenes que visitan el museo de manera independiente (Arriaga \& Aguirre, 2013). Junto a las actividades y contenidos para los jóvenes se agregan servicios adaptables que fomenten la socialización en el espacio del museo, como lo hace Los Angeles Museum of Contemporary Art (LACMA) con las actividades del programa Teen Event. After Dark en horarios nocturnos, inhabituales para una visita al museo pero cotidianos para las actividades sociales de los jóvenes.

La incorporación en los museos de las nuevas tecnologías de la información y comunicación tienen por finalidad conferir al público joven un papel activo en el desarrollo de la comunicación del museo (Stuedahl, 2011) y explorar nuevas museografías pedagógicas (Huerta, 2011). Con estas tecnologías se puede estimular el conocimiento recíproco entre museo y jóvenes así como el diálogo permanente, el que se formaliza en estrategias de comunicación adaptadas a este fin. Así lo realiza el Walker Art Center a través de su web o el blog y Facebook de jóvenes 
del Metropolitan Museum de Nueva York. Ambos usan las nuevas tecnologías para que el museo se dirija a los jóvenes informando sobre lo que hace, pero a la vez asume estas plataformas como un espejo que refleja lo que los jóvenes piensan, opinan y dialogan sobre el museo. Vale recordar que es justamente esta dimensión de realización tecnológica y sus repercusiones en la organización productiva y simbólica de la sociedad, junto al consumo cultural y el discurso jurídico los tres elementos que le dan sentido y especificidad al mundo juvenil más allá de los límites biológicos de la edad (Reguillo 2007).

\section{Zapping y tiempo detenido: encuentros/desencuentros de los jóvenes con el museo}

En el contexto de la investigación reseñada, las percepciones y argumentos de las y los jóvenes chilenos sobre los museos se exploraron a partir de seis focus groups, en el rango etario de 18-25 años de edad, divididos en dos conjuntos: con jóvenes que se reconocían público de museo y el segundo con jóvenes no-público de museo. La homogeneidad de los grupos radicó en el autoreconocimiento como público o no público de museos.

Dado que los objetivos de los grupos focales consisten en identificar cuestiones radicadas en la subjetividad de los sujetos, nos propusimos realizar grupos focales inspirado en la noción de Grupo de Discusión, de Jesús Ibáñez (Más allá de la sociología: el grupo de discusión, teoría y crítica, Siglo Veintiuno; Madrid; España. 5a. ed., 2003). Esta opción nos permitió abandonar el marco restrictivo del grupo focal como técnica de investigación, ya que este se orienta a la captura de respuestas eficientes para los objetivos del estudio. El grupo de discusión, por su parte, prioriza, como opción epistémica, la posibilidad de generar el habla social de un grupo sobre el tema estudiado, aunque sea en una situación artificial o de laboratorio. Esto permite ampliar el campo de observación, a diferencia del grupo focal que lo fragmenta y restringe.

Para los efectos de este estudio los "no usuarios" de museos corresponden a las personas que no han visitado ningún museo y por aquellas que, aunque no han visitado ninguno, estarían dispuestas a visitar alguno (Alcalde Gurt \& Rueda i Torres, 2008). Es cierta la dificultad de hallar personas que manifiesten una negativa radical a visitar museos, pero también es verdadero que hay gran cantidad de población sin ningún interés por conocerlos.

Los focus groups fueron heterogéneos desde el punto de vista del sexo, con 11 participantes en promedio (64 personas en total con los 6 grupos), todos estudiantes de nivel universitario- técnico y solo un estudiante secundario. Se ejecutaron en la Escuela de Periodismo de la Universidad de Santiago de Chile, en el Museo de Historia Natural de Concepción y en la Universidad Católica de Valparaíso, y se privilegió la moderación que permitiera la libre participación, con bajo control sobre la interacción de los participantes. Cada sesión duró aproximadamente 90 minutos a partir de un set de cinco preguntas iguales para público y no-público, y dos distintas (ver tabla 1). Se grabaron en audio, se transcribieron y codificaron a partir de categorías preliminares, seguido del reconocimiento de categorías y subcategorías emergentes, enunciadas por los propios participantes. 


\begin{tabular}{|c|c|c|}
\hline & No Público & Público \\
\hline 1 & \multicolumn{2}{|c|}{$\begin{array}{l}\text { ¿Recuerdan cuándo fue la primera vez que estuvieron o visitaron un museo y cómo } \\
\text { fue esa experiencia, quién los llevó, qué edad tenían? }\end{array}$} \\
\hline 2 & \multicolumn{2}{|c|}{ ¿Cuándo escuchan la palabra "museo" qué es lo primero que se les viene a la cabeza? } \\
\hline 3 & \multicolumn{2}{|c|}{$\begin{array}{l}\text { Según ustedes ¿qué importancia o función social tienen los museos en el Chile de } \\
\text { hoy? ¿Cuál sería la relevancia? }\end{array}$} \\
\hline 4 & \multicolumn{2}{|c|}{$\begin{array}{l}\text { Según ustedes ¿qué es lo que debería “dejar” la visita al museo?: conocimiento, } \\
\text { entretención, emoción... }\end{array}$} \\
\hline 5 & \multicolumn{2}{|c|}{$\begin{array}{l}\text { Según las estadísticas que se manejan hoy, los museos reciben menos visitas que el } \\
\text { cine, los conciertos y el teatro. ¿A qué creen que se debe eso? }\end{array}$} \\
\hline 6 & $\begin{array}{l}\text { ¿Qué es lo que impide que los } \\
\text { jóvenes vayan a los museos? }\end{array}$ & $\begin{array}{l}\text { ¿Qué es lo que creen que hace atractivo } \\
\text { a un museo para visitarlo? }\end{array}$ \\
\hline 7 & $\begin{array}{l}\text { ¿Qué es lo que deberían hacer los } \\
\text { museos para motivar las visitas de los } \\
\text { jóvenes? }\end{array}$ & $\begin{array}{l}\text { ¿Qué es lo que no hace atractivo a un } \\
\text { museo? }\end{array}$ \\
\hline
\end{tabular}

Tabla 1. Preguntas grupos focales jóvenes no público y público de museos.

Las percepciones compartidas entre los jóvenes que se reconocen como público y no-público de los museos coinciden en que los museos son en general instituciones relevantes en la sociedad, y cumplen la finalidad común de preservar objetos valiosos, especiales, únicos, sobre los que educan. Es por esta razón que los jóvenes consideran que no es comparable el museo con el resto de la oferta de consumo cultural, pues todo lo demás está orientado a proporcionar diversión y entretenimiento.

Pese a la valoración del museo como institución cultural plenamente vigente, los jóvenes coinciden en cuestionar la actuación de los museos chilenos en una variedad de planos como servicios de apoyo y mediación, difusión y comunicación, comisariado y museografía, y propuesta educativa. Destacan la escasa voluntad por informar sobre lo que hacen, abandono del visitante en las exposiciones, ausencia de novedad, sorpresa, diversidad; persistencia en las tradicionales restricciones como el "no tocar", "no cruce la línea", "silencio"; "sólo mire". Otras críticas residen en la falta de interactividad; insuficiente modernización; carencia de diversidad cultural en las muestras y oferta de experiencias poco estimulantes.

Se aprecia en sus reflexiones que el museo es el punto de observación para comparar la sociedad del pasado, remitida a sus percepciones en la infancia temprana, con la del presente, representada por su experiencia actual de jóvenes. El recuerdo de la primera visita al museo como actividad escolar fue una referencia reiterada entre los jóvenes, conducidos por un profesor junto a sus compañeros, en un museo de su localidad. 
Para los jóvenes público en particular hubo "algo", un objeto o pieza de la colección, un detalle del inmueble o característica del ambiente (silencio, iluminación, tamaño de la sala, temperatura ambiental) que llamó su atención infantil y marcó la memoria sobre la visita, la que luego repitió en reiteradas ocasiones con sus padres o familia. En tanto, los jóvenes no público rememoran la primera visita como una actividad escolar aburrida desde la que construyen un imaginario sobre el museo equivalente a la escuela o un espacio educativo:

No voy al museo porque paso todo el día estudiando y ¿cómo voy a ir a un lugar para seguir estudiando? Lo que quiero es desestresarme y divertirme (3:89).

La impronta educativa de los museos chilenos se revela con nitidez en las percepciones de todos los jóvenes, y deja al descubierto una perspectiva educacional basada en la transmisión de conocimiento replicando las finalidades de la escuela, y menos la estimulación del goce o fruición que reclaman los jóvenes, "vivir emociones". El mayor énfasis lo manifiestan los jóvenes público de museos que establecen comparaciones con museos extranjeros como Argentina, Perú y Europa, y además coinciden en que pertenecen a niveles socioeconómicos más elevados. En tanto, los jóvenes público de estratos socioeconómicos más bajos comparan con los museos de la capital.

Jóvenes no-públicos señalaron como anti ejemplo de museo al Museo Interactivo Mirador (MIM), un centro de interpretación de la ciencia y no un museo propiamente tal. Los jóvenes no-público preguntaron en el transcurso de las conversaciones si el MIM era considerado museo porque lo recordaron como una experiencia divertida, y contradictoria con los esperado en una visita escolar "siempre aburrida".

El tiempo apareció como otra categoría significativa en las percepciones de los jóvenes, la que se expresa en oposición a un pasado lento y continuo que ofrece el museo frente al presente rápido y fragmentado que viven los jóvenes. Para ellos el museo se debate entre el tiempo detenido y los recortes y rearmados de la vida zapping actual. Los jóvenes manipulando el control remoto de su cotidianidad.

Los museos en Chile uno los ve y los intuye como lugares en los que hay que pasear despacio, con cuidado, con silencio, o sea, es una experiencia de baja velocidad y lamentablemente todo y toda la sociedad es rápida. (1:153).

Además se observa como apreciación compartida el concepto restringido de cultura que persiste en los museos, referido fundamentalmente a la carencia de discurso crítico y reflexivo.

El museo trata de mostrarte todo bonito, pero lo actual como el arte u otras cosas, no son belleza, es mensaje, contestaciones, información, pero no estrictamente belleza $(2: 27)$.

La presencia constante del discurso del poder o hegemónico y el silencio sobre "la historia más cercana a la gente" (4:72) aparece como reflexión recurrente entre jóvenes público y no-público. 
En síntesis se establece entre las percepciones de los y las participantes elementos relacionados con aspectos del funcionamiento y los servicios del museo, así como conceptualizaciones sobre los contenidos y actividades que emprende. Las críticas no merman la valoración del museo como institución cultural plenamente vigente, sin embargo revelan los jóvenes la especificidad de su matriz cultural, referida -entre otros aspectos- a una temporalidad social basada en el fragmento que se opone al relato de continuidad al que apela la museografía tradicional imperante en Chile. El reclamo por un discurso cultural de la familiaridad, proximidad, que permita situar el relato del museo en su propio relato personal cotidiano. Por último, los jóvenes esperan reconocer en el museo la ruptura y la crítica en lugar de la armonía y acuerdo sobre las representaciones sociales que se presentan, en una experiencia estética/ histórica museal basada en el placer y la fruición. Estos podrían considerarse de manera preliminar como los ejes del "desencanto" de los jóvenes en el museo y los indicios de la constitución del objeto de estudio que debemos levantar para superar la incomprensión sobre estos actores sociales.

\section{Conclusiones}

La identificación de los elementos que determinan que la visita al museo sea una experiencia positiva y satisfactoria para los jóvenes exige mayor conocimiento de las prácticas culturales de este grupo en el espacio público. La visita al museo implica como primer esfuerzo salir de la casa y suspender por un rato las entretenciones que proporciona. La primera es apagar la televisión, la mayor competidora del museo y del resto de las actividades culturales (Barbero \& Morduchowicz, 2008). Salir de casa para ir al museo implica también dejar el computador, y optar por una actividad en la que el componente de socialización es tan relevante como el que podrían hallar en las redes sociales digitales. Para los jóvenes el componente socializador de la visita al museo ha sido completamente oscurecido por la finalidad educativa colectiva. En este sentido el argumento del desencanto de los jóvenes por los museos se relativiza y admite el de la incomprensión de sus prácticas culturales heterogéneas y omnívoras. El desarrollo de estudios que evalúen la eficiencia de las estrategias integrales de los museos para acercarse a los jóvenes y el conocimiento de las asociaciones y conexiones entre el consumo museal de este grupo etario con otras de sus prácticas culturales y sus representaciones simbólicas permitirá esbozar una suerte de modelo estructura que revele las modalidades de estas prácticas. Esta recomendación ya ha sido formulada en el escenario de los estudios sobre los "no lectores y "la poca lectura" (Bahloul, 1998), fenómeno que presenta aristas semejante al del consumo museal en tanto permite reconocer prácticas que responden a condiciones sociales que superan el nivel socioeconómico y educativo, como el estatus, género y rango de edad. 


\section{Referencias}

Alcalde Gurt, G. \& Rueda i Torres, J.M. (2008). Una aproximación al análisis del no-público de los museos a partir del estudio de uso del museo de arte de Girona. Mus-A. Revista de los Museos de Andalucía, (10), 90-95.

Almanza, V. (2005). Los Estudios sobre el Consumo Cultural: Algunas Observaciones Metodológicas. Revista Razón y Palabra, 47.

Antoine, C. (2012). La orientación hacia las audiencias en las políticas culturales. Venturas y desventuras de los incentivos públicos para aumentar el acceso a los bienes culturales. El caso de Chile. En: VICCI, G. (ed.) Seminario Públicos y Artes Escénicas. Montevideo: Teatro Solís - CIDDAE.

Ancel, P. \& Pessin, A. (2004). Les non-publics: les arts en réceptions, Paris.

Appadurai, A. (1988). The social life of things: Commodities in Cultural Perspective. Cambridge: Cambridge Universitiy Press.

Arriaga, A. \& Aguirre, I. (2013).The role of the learner in the construction of meaning at Tate Britain. Arte, Individuo y Sociedad, 25(2), 203-218. http://dx.doi. org/10.5209/rev_ARIS.2013.v25.n.2.42077

Asensio Brouard, M. ., \& Asenjo Vergara, E. (2011). Lazos de luz azul: museos y tecnologías 1, 2 y 3.0. Madrid. Editorial UOC.

Bahloul, J. (1998). Lecturas precarias. Estudio sociológico de los «poco lectores». México: Fondo de Cultura Económica.

Bastías Sekulovic, M. (2012). Museos y Públicos: Apuntes sobre una relación distante. Observatorio Cultural Valparaíso: Departamento de Estudios, Consejo Nacional de la Cultura y las Artes.

Barbero, J.M. (1999). Recepción de medios y consumo cultural: travesías. En Sunkel, G. (Ed.) El Consumo Cultural en América Latina: construcción teórica y líneas de investigación. (pp.47-71). Bogotá: Convenio Andrés Bello.

Barbero, J.M. \& Morduchowicz, R. (2008). Los jóvenes y las pantallas: nuevas formas de sociabilidad, Barcelona: Gedisa.

Baudelot, C.; Cartier, M. \& Détrez, C. (2010). Y sin embargo leen... Veracruz: Universidad Veracruzana.

Bazin, P. (2005). El fin del orden en la lectura. Medium, 4, 7-21.

Bennett, T. (2009). Museum, field, colony: colonial govermmentality and the circulation of reference. Journal of Cultural Economy, 2(1-2), 99-116. http:// dx.doi.org/10.1080/17530350903064097

Bermúdez, E. (2001). Consumo cultural y representación de identidades juveniles. Congreso LASA 2001. Washington DC.

Bialogorski, M. \& Cousillas, A. M. (2008). Una propuesta de evaluación innovadora en los estudios de visitantes de un museo de Buenos Aires. Mus-A. Revista de los Museos de Andalucía, 10, 98-101.

Bisbal, M. (1999). La idea del consumo cultural: teoría, perspectivas y propuestas. Comunicación, 108, 32-39.

Bouder-Pailler, D. \& Damak, L. (2009). Risque perçu, confiance et consommation de spectacles vivants : enjeux théoriques et managériaux. Paper presentado a la $10^{\circ}$ International Conference on Arts and Cultural Management (AIMAC 2009), Dallas, EE.UU. 
Bourdieu, P. (1998). La distinción. Crítica social del gusto. Madrid: Taurus.

Boylan, P. (2006). Museums: Targets or Instruments of Cultural Policies? Museum International, 58 (4), 8-12. http://dx.org/10.1111/j.1468-0033.2006.00577.x

Cari, C. (2012). Los visitantes a los museos que no están en las encuestas [Online]. Santiago: Magister en Gestión Cultural, Universidad de Chile. Available: http:// mgcuchile.cl/wp-content/uploads/downloads/2013/07/visitantesdelosmuseos.pdf [Acceso 26 de diciembre de 2013].

Catalán, C. (2009).Consumo y segmentación: Algunas consideraciones conceptuales y empiricas. Santiago: Expansiva, Universidad Diego Portales.

Certeau, M. (1996). La invención de lo cotidiano. México: Universidad Iberoamericana.

Consejo Nacional de la Cultura y la Artes (CNCA) (2007). Encuesta de Consumo Cultural 2004-2005. Valparaíso.

Consejo Nacional de la Cultura y la Artes (CNCA) (2009). 1ra. Encuesta Nacional de Participación y Consumo Cultural. Santiago: Ediciones Cultura.

Consejo Nacional de la Cultura y la Artes (CNCA) (2011). 2da. Encuesta Nacional de Participación y Consumo Cultural. Santiago: Ediciones Cultura.

Consejo Nacional de la Cultura y la Artes (CNCA) (2012). 3ra. Encuesta Nacional de Participación y Consumo Cultural. Santiago: Ediciones Cultura.

Cousillas, A.; Altamirano, C.; Cardinal, M.J.; Di Fiore, M. V. \& Ricci, C. R. (1998). Frecuentación y consumo cultural del público a museos. Aportes para repensar la política cultural de Buenos Aires. 1er Congreso Virtual de Antropología y Arqueología.

Chan, T.W \& Goldthorpe, J.H. (2010). Social Status and Cultural Consumption. En Chan, T.W (Ed.) (2010) Social Status and Cultural Consumption. (pp. 1-27). London: Cambridge University Press.

De la Iglesia, B., \& Roselló, R. (2014). Identificación de las barreras de acceso a la información, a la participación y el aprendizaje en el Museo Es Baluard. Arte, Individuo y Sociedad, 26(1), 21- 34.

Devos, F. (2006). Jóvenes pero alcanzables. Técnicas de marketing para acercar la cultura a los jóvenes. Madrid: Fundación Autor.

Domínguez, M. \& Sádaba, I. (2005). Transformaciones en las prácticas culturales de los jóvenes. De la lectura como ocio y consumo a la fragmentación neotecnológica. Revista de Estudios de Juventud, septiembre, 5(70); 23-38.

Douglas, M \& Isherwood, B. (1996). The World of Goods. Towards an anthropologist of consumption. London: Routledge.

Fernández Orgaz, L. (2002). Visitando un museo de arte con alumnos de educación infantil. Arte, Individuo y Sociedad, Anejo 1, 437- 440.

Gayo, M. (2013). La teoría del capital cultural y la participación cultural de los jóvenes. El caso chileno como ejemplo. Última Década (38), 141-171.

García Canclini, N. (1993). El consumo cultural en México. México DF: Grijalbo.

Gómez del Águila, L. (2011). Accesibilidad e inclusión en espacios de arte: ¿cómo materializar la utopía? Arte, Individuo y Sociedad, 24(1), 77-90. http://dx.doi. org/10.5209/rev_ARIS.2012.v24.n1.38044 
Gutiérrez del Castillo, R. (2010). Desarrollo de audiencias: fines y medios. Conectando Audiencias, 1 (1), 7-9.

Güell, P., Morales, R. \& Peters, T. (2011). Tipología de prácticas de consumo cultural en Chile a inicios del siglo XXI: mismas desigualdades, prácticas emergentes, nuevos desafíos. Revista Universum 2(26). http://dx.doi.org/10.4067/S071823762011000200007

Huerta, R. (2011). Maestros, museos y artes visuales. Construyendo un imaginario educativo. Arte, Individuo y Sociedad, 23(1), 55-73. http://dx.doi.org/10.5209/ rev ARIS.2011.v23.n1.5

Instituto Nacional de Estadísticas (INE). (2010). Cultura y tiempo libre: Informe Anual 2003-2009. Santiago: INE.

Jones, C. (2012). La tarjeta de lealtad del Chapter. Una idea originaria de los supermercados. Conectando Audiencias 6, 12-15.

Kirchberg, V. (1996). Museum visitors and non-visitors in Germany: A representative survey. Poetics, 24(2-4), 239-258.

Kumar Warikoo, N. (2011). Balancing acts: youth culture in the global city. Berkeley: University of California Press.

Laboratorio Permanente de Públicos de Museos (2010). Conociendo a nuestros visitantes. Estudio de público en museos. Madrid: Ministerio de la Cultura de España.

Lekue, P. (2009). Actitudes del alumnado preadolescente hacia la enseñanza artística en el museo: la experiencia del Museo Artium de Vitoria/Gasteiz con centros educativos públicos y concertados de las comunidades autónomas vasca y navarra. Ikastorratza e-Revista de didáctica (5), 1-7.

Mengin, A. D. (1993). La recherche d'une typologie des publics à la Cité des Sciences et de l'Industrie. Publics et Musées, 47-65.

McCracken, G. (1990). Culture and Consumption. Bloomington: Indiana University Press.

Obregón Fernández, A. (2011). Estudio de los recursos educativos para público familiar en los museos de arte europeos y norteamericanos. Arte, Individuo y Sociedad, 24(1), 9-20. http://dx.doi.org/10.5209/rev_ARIS.2012.v24.n1.38040

Observatorio Cultural (2011). Museos. Reporte estadístico $N^{\circ}$ 5. Valparaíso: Consejo Nacional de la Cultura y las Artes (CNCA).

Peters, T. (2010). La afinidad electiva entre consumo cultural y percepción sociocultural: el caso de Chile. Signo y Pensamiento, 29(57), 216-235.

Poulain, M. (2004). Entre preocupaciones sociales e investigación científica: el desarrollo de sociologías de la lectura en Francia en el siglo XX. En Lahire, B. (Ed.) (2004) Sociología de la lectura: del consumo cultural a las formas de la experiencia literaria. (pp. 17-58). Barcelona: Gedisa.

Programa de Naciones Unidas para el Desarrollo (PNUD). (2002). Desarrollo humano en Chile. Santiago: PNUD

Revista Patrimonio Cultural (RPC) (2005). Museos: disculpe las molestas...estamos trabajando para usted. Revista Patrimonio Cultural-DIBAM 10(37), 28-30.

Reguillo, R. (2000). Emergencia de culturas juveniles. Estrategias del desencanto, Bogotá: Norma. 
Reguillo, R. (2007). Las culturas juveniles: un campo de estudio; breve agenda para la discusión. En Fávero, O.; Pontes, M; Carrano, P. \& Reys R. (Eds.) Juventude e Contemporaneidade. (pp. 47-72). Brasilia: Ministério da Educação.

Rey, G. (2008). Las tramas de la cultura. Bogotá: Secretaría General Convenio Andrés Bello.

Rosas Mantecón, A. (2005). Usos y desusos del patrimonio cultural: retos para la inclusión social en la ciudad de México. Anais de Museu Paulista. Universidad de Sao Paulo, 13, 235-256.

Savage, M. \& Gayo, M (2011). Unravelling the Omnivore: a field analysis of Contemporary musical taste in the United Kingdom. Poetics (39). http://dx.doi. org/10.1016/j.poetic.2011.07.001

Schouten, F. (1998). Professional and visitors: closing the gap. Museum International. UNESCO, 50, 27-30. http://dx.doi.org/10.1111/1468-0033.00173

Storey, J. (1999). Cultural consumption and everyday life. Bloomsbury Academic.

Stuedahl, D. (2011). Young Visitors. Messing Around in Museums. Exploring social media to engage teens in participation. Norwegian Centre for Child Research (34), 169 - 191.

Sunkel, G. (2002). Una mirada otra. La cultura desde el consumo. En Mato, D. (Ed.) Estudios y otras prácticas intelectuales latinoamericanas en cultura y poder. (pp.102-113). Caracas: Consejo Latinoamericano de Ciencias Sociales (CLACSO).

Torche, F. (2007). Social Status and Cultural Consumption: The Case of Reading in Chile. Poetics 35 (2-3), 70-92. http://dx.doi.org/10.1016/j.poetic.2007.03.004

Torche, F. (2010). Social status and public cultural consumption: Chile in comparative. En Chang, T. (Ed.) Social Status and Cultural Consumption. (pp.109-138). London: Cambridge University Press.

Wallace, M. (2006). Museum Branding: How to Create and Maintain Image, Loyalty, and Support. Rowman Altamira.

Xanthoudaki, M. (1998). Educational Provision for Young People as Independent Visitors to Art Museums and Galleries: Issues of Learning and Training. Museum Management and Curatorship, 17 (2), 159-172. http://dx.doi. org/10.1080/09647779800401702 